\title{
The Nature of Transient Dark States in a Photoactivatable Fluorescent Protein
}

\author{
Arijit Roy, ${ }^{+, \perp}$ Martin J. Field, ${ }^{*,+}$ Virgile Adam, ${ }^{\neq, \|}$and Dominique Bourgeois ${ }^{*,+, \S}$ \\ ${ }^{\dagger}$ Institut de Biologie Structurale (IBS) —-Jean-Pierre Ebel, CEA/CNRS/Université Joseph Fourier, 41, rue Jules Horowitz, \\ 38027 Grenoble Cedex 1, France \\ ${ }^{\ddagger}$ Laboratory of Photochemistry and Spectroscopy, Department of Chemistry, Katholieke Universiteit Leuven, Celestijnenlaan 200F, \\ 3001 Heverlee, Belgium \\ ${ }^{\S}$ Pixel Team, IBS/Institut de Recherches en Technologies et Sciences pour le Vivant, iRTSV, Laboratoire de Physiologie Cellulaire \\ et Végétale, CNRS/CEA/INRA/Université Joseph Fourier, 38054 Grenoble, France
}

Supporting Information

ABSTRACT: Fluorescent proteins (FPs) of the green fluorescent protein family blink and bleach like all fluorophores. However, contrary to organic dyes, the mechanisms by which transient losses of fluorescence occur in FPs have received little attention. Here, we focus on the photoactivatable IrisFP, for which a transient non-fluorescent chromophoric state with distorted geometry was recently reported (Adam, V.; et al. J. Am. Chem. Soc. 2009, $131,18063)$. We investigated the chemical nature of this blinked state by employing quantum chemical/molecular mechanical calculations. Our findings suggest two previously unidentified dark states that display similar distorted chromophores with a transiently ruptured $\pi$ electron system. Both are protonated at atom $\mathrm{C}_{\alpha}$ of the chromophore methylene bridge. Transient protonation may occur via proton transfer from the nearby Arg66 either in the triplet state $T_{1}$ after intersystem crossing or in an anionic radical (doublet) ground state. As Arg66 is conserved in green-to-red photoconvertible FPs, these dark states are predicted to be common to all these proteins. We also suggest that $\mathrm{C}_{\alpha}$ protonated dark states may accelerate photobleaching by favoring decarboxylation of the fully conserved Glu212.

Tn the past few years, fluorescent proteins (FPs) have found ever Lincreasing use as markers in cell biology. Upon illumination, all FPs undergo transient stochastic switching events to non-fluorescent dark states, a phenomenon known as "blinking". 1,2 Conversion to a permanent off state eventually occurs in a process known as "bleaching". ${ }^{1}$ Although they can be used to advantage in the design of, e.g., super-resolution microscopy schemes, blinking and bleaching are impediments to the utilization of FPs in most imaging applications. ${ }^{3}$ To engineer FPs in which these effects are better controlled, the underlying mechanisms must be understood. ${ }^{4}$

Blinking and bleaching encompass a variety of photochemical pathways. In organic dyes, blinking has been attributed to the generation of excited triplet states ${ }^{5-7}$ or the subsequent formation of chromophore radical species through electron transfer. ${ }^{8,9}$
Similar species may also be formed in FPs, as recently suggested by us ${ }^{10}$ and others, ${ }^{11}$ but their exact chemical nature remains obscure. How such species may accelerate bleaching or promote photoactivation, leading to covalent modification of the chromophore and/or its environment, through, for example, reaction with reactive oxygen species ${ }^{12}$ or side-chain decarboxylation ${ }^{13-16}$ is also unclear.

Recently, we observed a metastable dark state in the photoactivatable fluorescent protein IrisFP, ${ }^{17}$ using X-ray-induced solvent radiolysis as a source of electrons and holes, and cryogenic temperature. ${ }^{10}$ The transition between the fluorescent and dark forms was mostly reversible, with only a small irreversible component, and the rate of fluorescence recovery increased rapidly with temperature. ${ }^{10}$ The crystallographic data suggested a significantly distorted 4-( $p$-hydroxybenzylidene)-5-imidazolinone chromophore, with the loss of planarity occurring about the bridging $\mathrm{C}_{\alpha}=\mathrm{C}_{5}$ methylene group and resulting in a transient disruption of the conjugated $\pi$-electron system. (See Supporting Information (SI) Figure S1 for atom nomenclature.) In crystallo Raman spectra supported the finding, as it revealed a decrease of the $1545 \mathrm{~cm}^{-1}$ band, assigned in part to the methylene group. Although Raman spectra also indicated that a similar dark state could be formed under excitation with visible light, the nature and relevance of the X-ray-induced distorted chromophore remained to be established.

Here, by using computational approaches, we suggest that the distorted dark state in IrisFP could correspond to a chromophoric form protonated at atom $\mathrm{C}_{\alpha}$. We find that protonation followed by distortion is possible in the excited triplet state or in the ground doublet state after electron transfer, thus revealing two previously unidentified transient dark states. The duality of the mechanisms by which chromophore distortion can arise highlights the relevance of the experimental approach that we previously used. ${ }^{10}$

To identify the nature of the distorted dark state, we first refined a model of its structure from the previously obtained crystallographic data. $^{10}$ (See the SI for details and Figure S2.) The model reveals that, in the distorted state, the $\mathrm{C}_{\alpha}$ atom of the chromophore approaches the Arg66 guanidinium moiety at a distance of $\sim 3.0 \AA$, suggesting a potential interaction between

Received: September 9, 2011

Published: October 31, 2011 


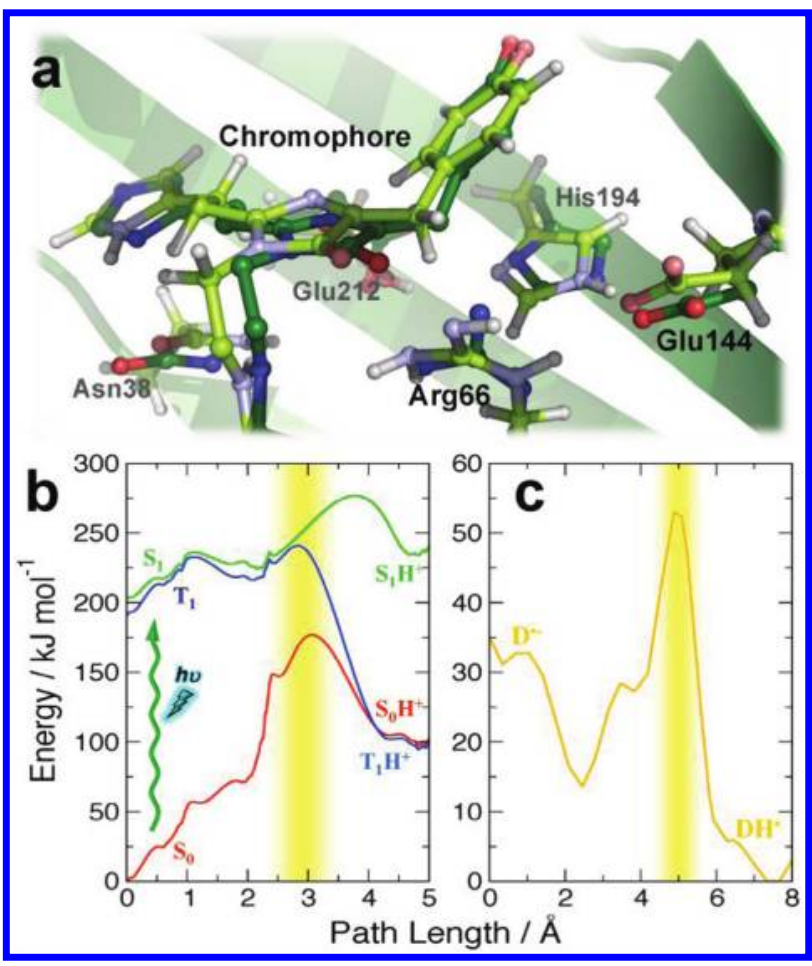

Figure 1. (a) Superposition of the chromophores of the experimental distorted (dark green carbons) and the average simulated $\mathrm{C}_{\alpha}$-protonated doublet (lime green carbons) structures. $(b, c)$ QC/MM energy profiles for proton transfer from Arg66 to the methylene bridge of the chromophore in IrisFP: singlet ground state, red; singlet excited state, green; triplet state, blue; doublet state with one extra electron, orange. The proton-transfer events occur within the ranges (b) 2.5-3.5 and (c) 4.5-5.5 A (yellow bands).

the two groups. We then performed extensive quantum chemi$\mathrm{cal} /$ molecular mechanical (QC/MM) calculations in conjunction with reaction-path-finding techniques and molecular dynamics (MD) simulations. (See the SI for details.) In line with the suggestion that electron transfer could be the cause of distortion, ${ }^{10,18}$ we first examined different charge (radical) states of the chromophore in both the ground and excited states, and assuming its phenolate form. However, all such calculations resulted in chromophores of planar or only weakly distorted geometry. By contrast, distorted structures comparable to that observed experimentally were obtained for various charge states when the $\mathrm{C}_{\alpha}$ atom of the phenolate chromophore was protonated and had $\mathrm{sp}^{3}$ hybridization (Figure 1a). The immediate environment of the $\mathrm{C}_{\alpha}$ atom provides very few possibilities for the source of the proton, but our calculations showed that Arg66 is a viable candidate. We identified two possible routes for the proton transfer, depending upon the charge state of the chromophore. The first route starts with excitation of the ground-state singlet chromophore $S_{0}$ to the $S_{1}$ state and intersystem crossing to $T_{1}$, and then involves proton transfer in the triplet state to $\mathrm{T}_{1} \mathrm{H}^{+}$, possibly followed by crossing back to a protonated $\mathrm{S}_{0} \mathrm{H}^{+}$ground state. Overall, the process is almost barrierless (after excitation) and endergonic, and results in a $\mathrm{C}_{\alpha}$-protonated chromophore that is separated from the planar form by a barrier of $\sim 80 \mathrm{~kJ} \mathrm{~mol}^{-1}$ (Figures $1 \mathrm{~b}$ and S3). The second route requires a doublet, in which one electron has been added to the system. It takes place in the ground state with a barrier of $\sim 40 \mathrm{~kJ} \mathrm{~mol}^{-1}$ and is exergonic
(Figure 1c). This route could be promoted by optical excitation followed by electron transfer in the $S_{1}$ or $T_{1}$ excited states and relaxation to the ground radical state $\mathrm{D}^{\bullet-}$, as observed in a number of organic dyes, ${ }^{8}$ and as substantiated by the growing evidence that FPs are implicated in a number of photoinduced electron-transfer mechanisms. ${ }^{11,14-16,19-21}$ It may also occur directly from the $S_{0}$ ground state, either by $\mathrm{X}$-ray-induced electron transfer or possibly in the presence of strong reducing agents. ${ }^{22}$ For both routes, the average values of the dihedral angles about the methylene bridge calculated from the simulations are in very good agreement with those observed experimentally (Figure 1a and Table S3).

Analysis of the pathways shows that the $\mathrm{C}_{\alpha}$ atom has the largest radical character in the planar-form doublet and triplet states, but this shifts to the $\mathrm{C}_{5}$ atom of the imidazolinone ring upon protonation. Similarly, the extra negative charge in the doublet state with respect to the singlet state is concentrated on the $\mathrm{C}_{\alpha}$ atom in the planar form but on the imidazolinone ring atoms in the distorted chromophore. In addition, calculation shows that the energetics of proton transfer from Arg66 is intrinsically highly favorable in all the spin states examined, but this is offset in the protein by the weakening of some important electrostatic interactions, such as that between Arg66 and the neighboring Glu144. Nevertheless, nanosecond QC/MM MD simulations at $100 \mathrm{~K}$ confirmed the stability of the $\mathrm{C}_{\alpha}$-protonated states of the chromophore, at least on the time scale of the simulation, as no back proton transfer was observed.

It is difficult to obtain reliable values for the rates of the proton-transfer processes that emerge from our calculations. However, we estimated these rates using simple barrier model fits to the energy profiles (see the SI). At $100 \mathrm{~K}$, hydrogen tunneling dominates for all reactions with corrections to the classical rate of $>10^{5}$, although the only proton transfers with acceptable rate constants are those leading to formation of the distorted doublet $\left(\sim 1 \mathrm{~s}^{-1}\right)$ and triplet $\left(\sim 10^{4} \mathrm{~s}^{-1}\right)$ chromophores, respectively. At room temperature, tunneling is much less important although still significant, with corrections of $\sim 3$, and the estimated rates $\left(\sim 10^{3}-10^{6} \mathrm{~s}^{-1}\right)$ indicate that the following proton transfers should occur readily: in both directions for the doublet; to form the triplet $\mathrm{C}_{\alpha}$-protonated chromophore; and to dissociate the singlet $\mathrm{C}_{\alpha}$-protonated chromophore. We note that the predicted rates for proton transfer in $T_{1}$ are consistent with typical triplet-state lifetimes at both temperatures.

In our previous experiment, ${ }^{10}$ we also noticed non-reversible bleaching of IrisFP induced by X-rays. The associated crystallographic structure revealed decarboxylation of the conserved Glu212, similar to observations made under UV light and attributed to a photo-Kolbe reaction mechanism. ${ }^{13-16,23} \mathrm{Calcu}-$ lations on model systems suggested that decarboxylation could possibly be linked to formation of the distorted state. These confirmed that decarboxylation is likely to occur only when Glu212 is in its neutral doublet state and also showed that electron transfer from Glu212 to the singlet or triplet states of the $\mathrm{C}_{\alpha}$-protonated chromophore is only marginally energetically disfavored. It is thus conceivable that a biradical charge-transfer state $\left(\mathrm{DH}^{\bullet} / \mathrm{Glu} 212^{\circ}\right)$ could be stabilized in the protein, leading to decarboxylation, although we were unable to observe intraQC-region electron transfer in our simulations of either the $\mathrm{S}_{0} \mathrm{H}^{+}$ or $\mathrm{T}_{1} \mathrm{H}^{+}$systems. In contrast, decarboxylation pathways were identified with barriers of $\sim 70 \mathrm{~kJ} \mathrm{~mol}^{-1}$ when an electron had 


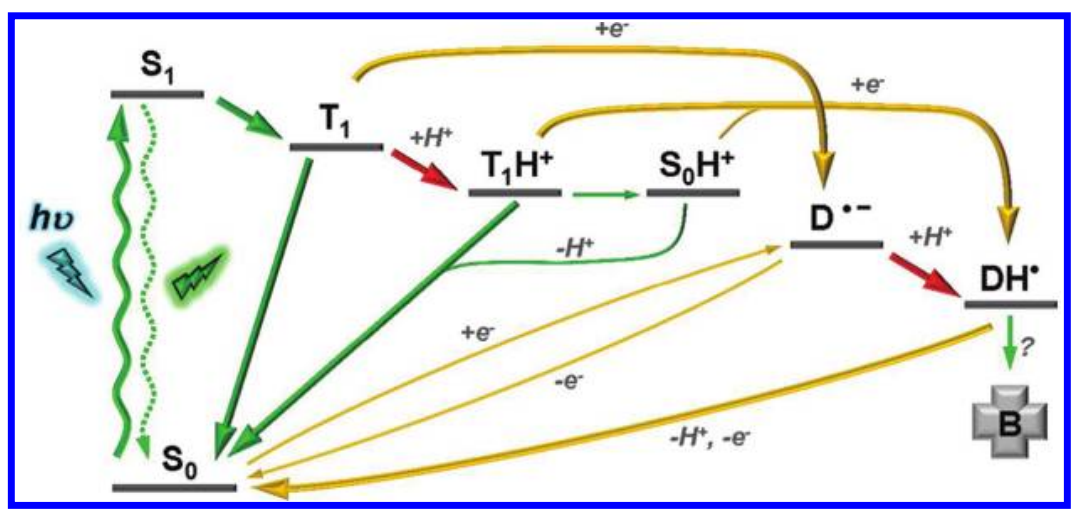

Figure 2. Proposed mechanism for transient dark-state generation in IrisFP. The thick orange and red arrows correspond to electron- and protontransfer steps in the visible light-induced reaction scheme, respectively, and the green arrows indicate other processes. The thinner arrows around the $\mathrm{S}_{0} \mathrm{H}^{+}$state are to emphasize the uncertainty that this state plays in the mechanism, which is dependent on its stability with respect to $\mathrm{T}_{1} \mathrm{H}^{+}$. The thinner orange arrows denote processes that are induced by X-rays or, possibly, by strong reducing agents, ${ }^{22}$ whereas the arrow with a question mark highlights a potential decarboxylation route. D, doublet; S, singlet; T, triplet; B, bleached.

been removed from the QC region (i.e., for systems in the $\mathrm{D}^{\bullet+}$ state). Generation of the distorted and bleached states in IrisFP may thus occur via different pathways. Interestingly, the results also suggest that, although not directly coupled, these two processes could be indirectly linked in the tetrameric or highly concentrated monomeric protein. (See the SI for further discussion.)

A schematic of the processes that we have presented above is given in Figure 2. As a test of generality, we did exploratory calculations of a number of other green-to-red photoconvertible FPs (EosFP, Dendra2, and Kaede) and obtained consistent results as long as there was a suitable proton donor close to the methylene carbon of the chromophore. As the presence of such a proton donor is a shared characteristic of many Anthozoan photoactivatable FPs, it would be interesting to study whether the protonation reactions described here also play a role in photoconversion or photoswitching mechanisms. ${ }^{24}$ We also note that Arg66 in IrisFP, or the equivalent residues in other photoconvertible FPs, could serve as a proton relay rather than as the main proton donor. In such a case, a proton donor more remote from the chromophore, such as His194 in IrisFP, could be implicated, which would result in further stabilization of the transient dark states (see the SI). Finally, although enhanced green fluorescent protein (EGFP) does not display a residue equivalent to Arg66, it is possible that a water molecule could play this role. As such, it would be interesting to investigate whether chromophore $\mathrm{C}_{\alpha}$-protonation upon electron transfer is involved in photooxidative ${ }^{11}$ or photoreductive ${ }^{19-21}$ EGFP redding or in Kolbe-like decarboxylation processes. ${ }^{13-16}$

In summary, we have investigated the nature of the recently reported transient dark state in IrisFP ${ }^{10}$ and conjecture that it is a chromophoric form in a doublet (radical) state protonated at atom $\mathrm{C}_{\alpha}$ of the methylene bridge. The importance of doublet states has been highlighted in the switching mechanisms of organic chromophores, and, interestingly, a concomitant proton transfer was recently suggested to take place in the ATTO655 synthetic dye. ${ }^{25} \mathrm{We}$ also find that $\mathrm{C}_{\alpha}$ protonation can occur in the triplet state $T_{1}$. In both cases a similar, severe distortion of the chromophore reversibly breaks the $\pi$-electron system and results in blinking. Such previously unsuspected protonation schemes may thus be quite general, may broaden our view of chromophore photophysics, and may lead to rational engineering of use in, for example, super-resolution microscopy.

\section{ASSOCIATED CONTENT}

S Supporting Information. Experimental and simulation methods and additional simulation results. This material is available free of charge via the Internet at http://pubs.acs.org.

\section{AUTHOR INFORMATION}

\section{Corresponding Author}

martin.field@ibs.fr; dominique.bourgeois@ibs.fr

\section{Present Addresses}

${ }^{\perp}$ Laufer Center for Physical and Quantitative Biology, Stony Brook University, Stony Brook, NY 11794-5252.

"Pixel Team, Institut de Biologie Structurale (IBS)-Jean Pierre Ebel, CEA/CNRS/Université Joseph Fourier, and Institut de Recherches en Technologies et Sciences pour le Vivant, iRTSV, Laboratoire de Physiologie Cellulaire et Végétale, CNRS/CEA/ INRA/Université Joseph Fourier

\section{ACKNOWLEDGMENT}

The French National Research Agency supported this work (grant ANR-07-BLAN-0107-01). The authors acknowledge the Centre de Calcul Recherche et Technologie in Paris and Pittsburgh Supercomputing Center for computer time, and Philippe Carpentier for initial refinement of the X-ray structure.

\section{REFERENCES}

(1) Dickson, R. M.; Cubitt, A. B.; Tsien, R. Y.; Moerner, W. E. Nature $1997,388,355$.

(2) Moerner, W. E. I. Chem. Phys. 2002, 117, 10925.

(3) Shaner, N. C.; Lin, M. Z.; McKeown, M. R.; Steinbach, P. A.; Hazelwood, K. L.; Davidson, M. W.; Tsien, R. Y. Nat. Methods 2008, 5,545 .

(4) Widengren, J.; Chmyrov, A.; Eggeling, C.; Lofdahl, P. A.; Seidel, C. A. I. Phvs. Chem. A 2007, 111, 429.

(5) Campos, L. A.; Liu, J.; Wang, X.; Ramanathan, R.; English, D. S.; Munoz, V. Nat. Methods 2011, 8, 143.

(6) Donnert, G.; Eggeling, C.; Hell, S. W. Photochem. Photobiol. Sci. 2009, 8, 481.

(7) Zondervan, R.; Kulzer, F.; Orlinskii, S. B.; Orrit, M. J. Phys. Chem. A 2003, 107, 6770. 
(8) van de Linde, S.; Krstic, I.; Prisner, T.; Doose, S.; Heilemann, M.; Sauer, M. Photochem. Photobiol. Sci. 2011, 10, 499.

(9) Vogelsang, J.; Kasper, R.; Steinhauer, C.; Person, B.; Heilemann, M.; Sauer, M.; Tinnefeld, P. Angew. Chem., Int. Ed. 2008, 47, 5465.

(10) Adam, V.; Carpentier, P.; Violot, S.; Lelimousin, M.; Darnault, C.; Nienhaus, G. U.; Bourgeois, D. I.Am. Chem. Soc. 2009, 131, 18063.

(11) Bogdanov, A. M.; Mishin, A. S.; Yampolsky, I. V.; Belousov, V. V.; Chudakov, D. M.; Subach, F. V.; Verkhusha, V. V.; Lukyanov, S.; Lukyanov, K. A. Nat. Chem. Biol. 2009, 5, 459.

(12) Jimenez-Banzo, A.; Nonell, S.; Hofkens, J.; Flors, C. Biophys. J. 2008, 94, 168.

(13) Bell, A. F.; Stoner-Ma, D.; Wachter, R. M.; Tonge, P. J. J. Am. Chem. Soc. 2003, 125, 6919.

(14) Henderson, J. N.; Gepshtein, R.; Heenan, J. R.; Kallio, K.; Huppert, D.; Remington, S. J. I. Am. Chem. Soc. 2009, 131, 4176.

(15) Subach, F. V.; Malashkevich, V. N.; Zencheck, W. D.; Xiao, H.; Filonov, G. S.; Almo, S. C.; Verkhusha, V. V. Proc. Natl. Acad. Sci. U.S.A. 2009, 106, 21097.

(16) van Thor, J. J.; Gensch, T.; Hellingwerf, K. J.; Johnson, L. N. Nat. Struct. Biol. 2002, 9, 37.

(17) Adam, V.; Lelimousin, M.; Boehme, S.; Desfonds, G.; Nienhaus, K.; Field, M. J.; Wiedenmann, J.; McSweeney, S.; Nienhaus, G. U.; Bourgeois, D. Proc. Natl. Acad. Sci. U.S.A. 2008, 105, 18343.

(18) Weber, W.; Helms, V.; McCammon, J. A.; Langhoff, P. W. Proc. Natl. Acad. Sci. U.S.A. 1999, 96, 6177.

(19) Elowitz, M. B.; Surette, M. G.; Wolf, P. E.; Stock, J.; Leibler, S. Curr. Biol. 1997, 7, 809 .

(20) Matsuda, A.; Shao, L.; Boulanger, J.; Kervrann, C.; Carlton, P. M.; Kner, P.; Agard, D.; Sedat, J. W. PLoS One 2010, 5, e12768.

(21) Sawin, K. E.; Nurse, P. Curr. Biol. 1997, 7, R606.

(22) Inouye, S.; Tsuji, F. I. FEBS Lett. 1994, 351, 211.

(23) van Thor, J. J.; Georgiev, G. Y.; Towrie, M.; Sage, J. T. J. Biol. Chem. 2005, 280, 33652.

(24) Dong, J.; Abulwerdi, F.; Baldridge, A.; Kowalik, J.; Solntsev, K. M.; Tolbert, L. M. I. Am. Chem. Soc. 2008, 130, 14096.

(25) Kottke, T.; van de Linde, S.; Sauer, M.; Kakorin, S.; Heilemann, M. I. Phvs. Chem. Lett. 2010, 1, 3156. 